\title{
Téoros
}

Revue de recherche en tourisme

\section{L'évolution de la formation en tourisme au Québec}

\section{André Bergeron, Pierre Durocher, Michèle L’Allier et Marc Laplante}

Volume 2, numéro 1, février 1983

Formation et conditions de travail en tourisme

URI : https://id.erudit.org/iderudit/1080838ar

DOI : https://doi.org/10.7202/1080838ar

Aller au sommaire du numéro

Éditeur(s)

Université du Québec à Montréal

ISSN

0712-8657 (imprimé)

1923-2705 (numérique)

Découvrir la revue

Citer cet article

Bergeron, A., Durocher, P., L’Allier, M. \& Laplante, M. (1983). L'évolution de la formation en tourisme au Québec. Téoros, 2(1), 6-13.

https://doi.org/10.7202/1080838ar d'utilisation que vous pouvez consulter en ligne. 


\title{
L'évolution de la formation en tourisme au Québec
}

\author{
par André Bergeron*
}

L'industrie du voyage et les divers secteurs relies au tourisme laccueil, animation, promotion, hótellerie, vente, amenagement, etc...) requièrent de plus en plus une maind'oeuvre qualifiée et dynamique au Québec en vue d'attirer et retenir chez nous le plus de visiteurs étrangers possible, pour valoriser aussi nos équipements et inciter nos concitovens à visiter d"abord leur coin de pays.

"C'est beau, c'est grand, c'est à voir", mais il faut aussi une main-d'oeuvre à la hau teur de nos aspirations et de nos defis.

L'accueil et l'hospitalité chez nous sont proverbiaux, du moins dans certaines régions bien spécifiques du Québec. Mais on peut taire davantage. Sans copier nos cousins français ou nos voisins americains, il y a place pour l'amélioration.

II ne s'agit pas uniquement de posséder de beaux paysages, des montagnes skiables. des rochers percés ou des portes St Jean, des iles d'Orléans, aux Coudres ou Ste Hélène, il faut aussi une main-d'oeuvre qua lifiée, dynamique, cultivée, polyvalente.

Qu'en est-il de cette formation de la main d'oeuvre en tourisme?

\section{Un peu d"histoire}

Nous devons d'abord bien distinguer tourisme et hótellerie. Ces deux programmes de formation sont bien differrents. L'Institut de tourisme et d'hötellerie du Québec offre les trois programmes disponibles au niveau collegial tandis que les cegeps de Granby et de Matane n'offrent que le programme des techniques du tourisme. Le Collège LaSalle offre le programme de tourisme axé sur la formation d'agent de voyage, en plus d'un programme en hótellerie. tandis que I'Université du Québec à Montréal prépare a "l"administration touristique" essen. tiellement.

Enfin d'autres universites "touchent" au tourisme de près ou de loin, certains centres

- Phere Durochet, de HIT.HO Mac Lablane de ruQAM et

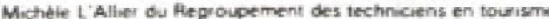

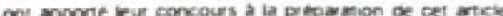

de formation en tourisme prives forment des agents de voyage assez rapidement et certaines commissions scolaires forment des guides-accompagnateurs.

Historiquement parlant, on a commencé à enseigner l'hôtellerie à partir de 1968 .

La creation de l'Institut de tourisme et d'hótellerie du Québec, par artêté ministériel en date du 17 juillet 1968, sous l'autorité du ministère du Tourisme, de la Chasse et de la Pêche Imaintenant du ministère de I'Industrie, Commerce et Tourismel, répondait à un double besoin de perfectionnement et de formation du personnel et de la maind'oeuvre appartenant au milieu du tourisme, de la restauration et de l'hótellerie du Québec.

II s'agissait, d'une part, de donner une qua lification professionnelle au personnel déa en place par l'entremise de cours de recyclage et de perfectionnement, et, d'autre part, d'être en mesure de former un personnel et une main-d'oeuvre spécialisés pour cette industrie touristique promise à un essor et à un développement remarquables au cours des décennies à venir.

L'enseignement du tourisme comme tel, au niveau collégial, remonte au début de la creation des Cegeps. En 1968, le nouveau Cegep de Granby se cherchait une vocation particulière. On songea alors à ne option speciale "tourisme" qui allait mettre le collège sur la carte du Québec. On fit appel à l'époque à un spécialiste du tourisme. Rejean Beaudoin, pour coordonner la mise sur pied de cette nouvelle option au Québec.

En 1969, avec l'appui de pressions politiques et administratives, le Cegep de Granby obtint la permission du ministère de l'Education d'experimenter un nouveau pro gramme. II s'agissait d'une année de spécialisation en tourisme après deux années de techniques administratives. II y eut quatre groupes de diplômés en techniques administratives, avec option tourisme, de 1972 à 1975 lannées de "promotion")

Déja le Cegep de Matane offrait, à parti de 1974, ce programme aux adultes dans un but de recyclage et de spécialisation de la main-d'oeuvre du Bas-St-Laurent/Gaspésie, le tout subventionne par le gouvernement féderal. Le Collège La Salle offrit aussi à partir de 1974 cette option expèrimentée à Granby.

II faut noter que durant ces quatre ou cing premières années, il y eut un comité de coordination pédagogique formé d'enseignants en tourisme et en loisir ainsi que de représentants de quelques secteurs de l'industrie touristique (ministère du Tourisme, de la Chasse et de la Pèche, agence de voyage, attraction touristique, etc...): ce comite recommanda à la Direction des études collégiales du ministère de l'Education, l'acceptation d'une nouvelle grille de cours correspondant à un nouveau programme de "techniques touristiques" en trois ans.

Ce nouveau programme fut accepté et officialisé à partir de septembre 1975. En méme temps on a permis, suite aussi à d'autres pressions politiques et administratives, à I'Institut de tourisme et d'hôtellerie du Québec d'offrir ce programme en tourisme, de mème qu'au Cegep de Matane pour ses étudiants reguliers, de jour

Plusieurs autres cegeps du Québec auraient bien voulu avoir cette option: XavierGarneau à Québec, ceux de Hull, de Rivièredu-Loup, et de Chicoutimi entre autres; mais les comités pédagogiques recommandaient à chaque annee au ministere de l'Education de limiter à quatre les collèges offrant ce programme, compte tenu des débouchés dans l'industrie touristique pour tous les finissants. La moitié seulement des finissants se trouvaient un emploi en tourisme.

Face à l'évolution des besoins des divers secteurs du tourisme lau moment du Sommet touristique de Sherbrooke, au moment de la création des associations touristiques régionales, entre autres), face aussi aux besoins et aux objectifs légèrement diffé rents au niveau de la formation de la part de chacune des maisons d'enseignement. compte tenu enfin quelquefois de contraintes budgétaires ou administratives, le comité de coordination pedagogique recommanda un nouveau programme de formation en tourisme à partir de septembre 1980 , celui qui est en application maintenant. 
Au niveau universitaire, il y eut toujours au Quẹbec un certain enseignement en tourisme; mais ce fut toujours organise de facon sporadique et ponctuelle, selon l'intérét et la motivation d'individus. Des exemples. Les departements de géographie de l'Universite de Montreal, de I'UQAM, et de Sherbrooke. oftraient dejaja il y a une dizaine d'annees des cours au niveau du baccalauréat, la plupart du temps des cours optionnels. Une dizaine d'etudiants, une vingtaine tout au plus, $s^{\prime} y$ sont spécialisés au niveau de la maittrise, y faisant quelques seminaires et recherches avant de soutenir leur these appliquee au tourisme.

L'Université Laval forme quelques économistes ayant le tourisme comme theme d'application. L'Université McGill forme quelques administrateurs. Les Universités du Québec a Trois-Rivières et celle d'Ottawa forment des récréologues qui s'intéressent aussi au phẻnomène touristique. L'Univer sité du Québec à Chicoutimi s'intéresse plus particulierement au tourisme social.

Seule I'UOAM a officiellement un "module de gestion et intervention touristiques", au niveau du baccalaureat, depuis 1978 dont les objectifs sont les suivants:

\section{Former des professionnels de la gestion} touristique

Si le tourisme, comme tel, semble difficile ment gérable, il donne lieu au développe. ment de nombreux services (transport, hébergement, restauration, animation, etc.l dont l'administration est particuliere; de plus, le tourisme exige des amenagements (naturels, culturels, sociaux), dont la planification et la gérance sont spécialisees: bases de plein air, sites naturels, lacs et rivières, lieux historiques, par exemple; enfin, le tou. risme est affaire de promotion et de publicité et la mise en marchè de ses produits relève d"une formation en gestion touristique.

\section{Former des agents de developpement en tourisme}

Si la formation professionnelie en gestion est dominante au "baccalaureat en tourisme" de I'UQAM, elle n'est ni exclusive, ni étroite. Le module a choisi d'être polyva lent et multi-disciplinaire. L'intervention en tourisme, deborde l'action administrative. Le tourisme est autant un phenomène socioculturel et socio-politique qu'une realite economique. Conséquemment, la formation offerte à rUQAM comprend de bonnes sensibilisations aux sciences humaines et socia les du tourisme, du loisir et du temps libre. et des cours specialises en tourisme social et en tourisme culturel. Enfin, une autre par. tie du programme se propose d'outiller le futur diplömé en tourisme d'une formation en recherche, en législation, en strategie d'action, bref par un entrainement à penser et à agir délibérément et respectant la glo balité et la complexité du domaine touristique.

\section{Former des animateurs de lidentite} culturelle québécoise
Culturellement, le tourisme peut devenif un phenomene d alienation culturelle et les etudes sur l'impact socio-culturel du tourisme dans les pays en voie de développement, par exemple, montrent que ce danger r'a rien d'une chimere. Le programme d'etudes en tourisme de I'UQAM s'occupe en priorite des questions relatives au tourisme $\mathrm{d}$ ac cueil (ou receptif): la mise en valeur des res sources naturelles et culturelles du Québec et de notre patrimoine, l'affirmation de nos diversites culturelles régionales et de nos facons propres de penser et de vivre, l'amelioration constante, donc, de nos fonetions d'hôte pour faire de la société québecoise une destination touristique de premier choix.

Cette orientation se manifeste par la pré sence de certains cours spécialisés sut ces sujets dans le programme, par la politique des stages de module et par le choix des engagements dans le milieu fait par les professeurs et les étudiants.

\section{Les programmes de formation en tourisme}

Au niveau collégial

Les techniciens en tourisme

Le technicien en tourisme est un praticien qui connait le phenomène touristique au niveau des différentes approches Idimen. sions economiques, sociales, géographiques, culturelles et administratives) et qui intervient dans les divers secteurs du tourisme, en favorisant et en participant à l'or: ganisation des deplacements et des séjours.

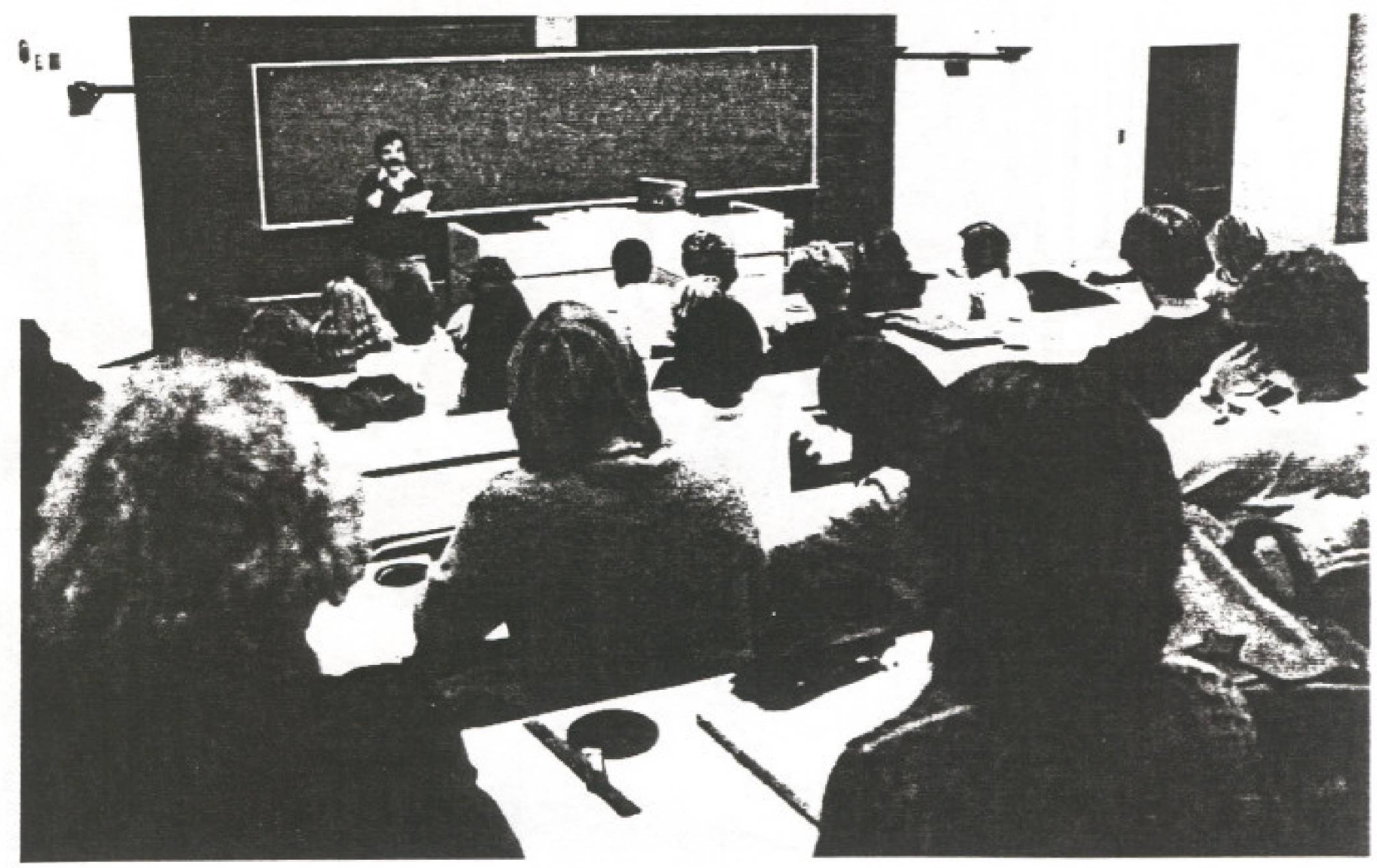


de personnes hors de leur horizon habituel à des fins de loisirs; c'est un agent de développement touristique: un organisateur, un promoteur, un animateur d'activités touris. tiques diverses, tant aux niveaux national que régional ou municipal, soit pour un orga nisme ou une entreprise touristique.

Les secteurs où il peut intervenir sont de dimension econamique et de dimension sociale. Dans la dimension économique, il intervient dans des agences de voyage, compagnies de transport, grossistes, hôtels, associations touristiques regionales, bureaux municipaux du tourisme, etc... Dans la dimension sociale, il peut intervenir dans des associations à but non lucratif telles que les Agricotours, les bases de plein-air, VeloQuébec, dans des mouvements coopératifs. dans des comités d'entreprises, etc... La maitrise du savoir, du savoir-faire et du savoir-être permet l'accession à des postes décisionnels plus élevés. On y privilégie un tourisme réceptif au Québec sans pour autant exclure le tourisme émetteur, du moins dans trois des quatre institutions qui offrent le cours (Granby, Matane, Lasalle).

\section{Les fonctions-types d'intervention}

du technicien en tourisme.

1) Agent de développement en tourisme

- Coordonnateur des services de tourisme:

- Agent de developpement:

- Agent de promotion;

- Agent de publicité:

- Organisateur de congres:
- Organisateur / Animateur de festivals populaires.

Soit pour une entreprise ou un organisme, tant aux niveaux régional que municipal.

2) Prépose à l'accueil

- Préposé á l'accueil et a l'information:

- Commis aux renseignements.

- Hotesses d'accueil.

Encore là, tant aux niveaux provincial que régional ou municipal; soit pour une entreprise privée ou un organisme public.

3) Agent de voyages et grossiste en voyages.

- Commis à la réservation.

- Conseiller en voyages:

- Billetiste:

- Forfaitiste

- Organisateur de voyages:

- Représentant (grossistel

4) Guide-accompagnateur

- Guide touristique:

- Guide-interprète;

- Courrier accompagnateur-animateur:

- Agent de transferts (compagnies de transportl.

Dépendamment de l'importance de l'entreprise, ces tâches peuvent être soit exclusi ves ou cumulatives.

Les écoles de formation

et les programmes

Quatre institutions ideux cegeps, un insti tut qui relève directement du ministère de l'Industrie, Commerce et Tourisme et un col lège privêl sont autorisès par le ministère de I'Education à dispenser le programme des techniques du tourisme au Québec: les cegeps de Granby et de Matane, l'Institut de tourisme et d'hótellerie du Québec et le Collège LaSalle.

Le cours est d'une durée de trois ans isix sessionsl et méne normalement et directement au marché du travail dans un des divers secteurs du tourisme.

En première année figurent des cours de formation generale (français, philosophie, mathematiques, langues, etc...), des cours d'introduction au tourisme, d'administration, et une première demarche de la connais. sance du milieu touristique. La deuxième année est consacree plus intensement aे la connaissance de techniques et d'outils ainsi qu'a lapprotondissement des connaissan" ces du milieu et des intervenants touristiques. La troisième année permet d'acqué fit d'autres techniques complementaires et d'intégrer toutes ces connaissances et techniques préalablement acquises. Le tout se fait idéalement lors d'interventions en milieu touristique: excursions, organisation de voyages, stage, préparation et présentation du projet touristique, etc.

Les cours offerts, en plus des cours obliga toires de formation fondamentale tels que langue et littérature, philosophie, éducation physique et mathematiques, sont essentiel. lement des cours relies au tourime comme:

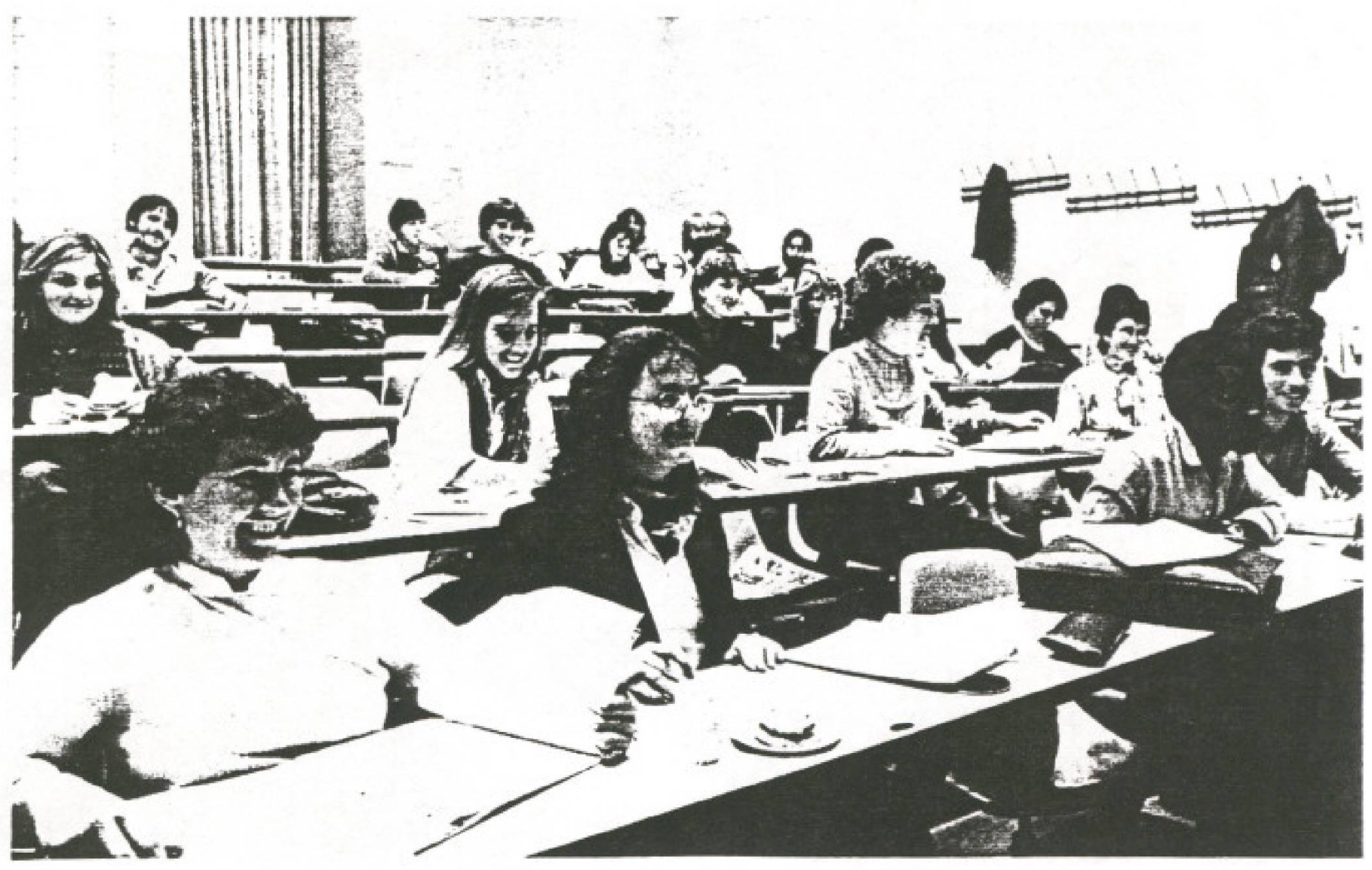


la socio-economie du tourisme, le tourisme régional au Quebec, les techniques d'ac cueil, les équipements touristiques, I'amé nagement touristique, les grandes attractions touristiques du monde, l'agence de voyage, l'organisation de voyage et l'orga nisation hótelière, ia législation touristique, la promotion touristique, sans compter un important projet touristique à èlaborer en fin d'ètudes.

On y recoit obligatoirement aussi des cours d'appoint; de statistique, d'administration. de comptabilite, de recherche commerciale de marketing et d'intormatique.

II faut ajouter, enfin, que la tendance est a la spécialisation. En effet, depuis deux ans chacune des institutions est autorisee à offri huit ou neuf cours qu'on retrouve dans une "banque de cours optionnels", dont voici quelques exemples: géographie lcarte du mondel. psychologie du touriste, économie du tourisme, grandes destinations touristiques du monde, tarification, excursions dans l'industrie (guidage), amenagement touris tique, les loisirs touristiques, la vente de services touristiques, l'organisation de congrès. l'agrotourisme, etc. Sans compter des cours d'anglais et d'espagnol.

Comme on le voit, cette formation qui se veut spécialisée est en fait globaliste sur le phenomene touristique. L'industrie touristique a besoin de specialistes certes, mais il semble quion forme des generalistes du tou. fisme, quitte à laisser aux emploveurs le soin de terminer sur place la formation necessaire et specifique.

\title{
La formation en hôtellerie
}

\author{
La formation en hốtellerie est bien différente de la formation en tourisme. \\ Dispensée à l'/nstitut de tourisme et d'hôtellerie du Québec. \\ cette formation s'articule autour de quatre axes: la gestion hôtelière, \\ la gestion des services alimentaires, la cuisine professionnelle, \\ la pâtisserie-boulangerie, le service de restaurant.
}

\section{La gestion hôtelière}

L'élève diplömé de ce cours pourra éven. tuellement occuper des emplois à tous les niveaux, compte tenu de ses qualifications et de l'expérience. Le champ d'activite du technicien en hôtellerie est étendu. II cou. vre des domaines variés et multidisciplinai. res, dont:

- I'hébergement: accueil, caisse reserva. tion, verification de nuit:

- I'entretien: planification, supervision et contrôle:

- la restauration: coordination et contrôle des salles a manger, des restaurants et des bars:

- le contrôle des différents départements: hébergement, service en général, prépa ration des aliments, personnel et entretien:

- la commercialisation hốteliere: établisse ment et mise en application des programmes de publicité

II peut accéder au niveau des cadres intermédiaires dans les domaines suivants: exploitation hôtelière, relations publiques. organisation des loisirs, publicité, compta bilité, gestion des differents services (production, hébergement, ventes, personnel, comptabilité et restauration.

\section{La gestion des services \\ alimentaires}

L'élève diplômé de ce cours será apte, après avoir acquis l'expérience nécessaire dans le milieu, à occuper un poste de responsable de la production alimentaire, de la formation du personnel.

Dans les hồtels, les restaurants, les cafété rias commerciales, industrielles ou d'institutions (hoppitaux, écoles, maisons d'accueill) pour les compagnies de services alimentai- res, les traiteurs, les compagnies de transport laériennes. maritimes et ferroviairesl, ou dans les secteurs de l'enseignement.

Le technicien en gestion des services ali. mentaires travaille sous la direction d'un chef de cuisine ou d'un responsable des ser: vices alimentaires avant d'accéder à ces pos tes après quelques années d'expérience.

Avant d'occuper un de ces postes, il doit maitriser le travail de la cuisine professionnelle, soit la transformation, la production et le controle des aliments; if doit egalement se familiariser avec l'approvisionnement et le stockage des marchandises. Les fonctions de responsable des services alimentaires exigent, outre ces connaissances essentieiles en cuisine, des solides connaissances en gestion de cuisine lapprovisionnement, production alimentaire qualitative et quantitative, ellaboration des menus, application des règles d'hygiène et de propreté), en gestion du personnel et en gestion budgétaire.

\section{La cuisine professionnelle}

Le cours de cuisine professionnelle a pour but de former des candidats aptes à occuper un poste de cuisinier dans les differrents etablissements d'accueil tels que hôtels, restaurants, cafétérias industrielles et hôpitaux

Selon l'endroit out il travaille, il peut occu per un ou plusieurs des postes suivants.

- garde-manger; cuisinier qui prépare les hors-d'oeuvre et les plats froids, et qui a la responsabilité de la conservation et du detaillage des pieces de boucherie. des poissons, etc.:

- saucier: cuisinier qui apprête tous les mets en sauce:

- entremettier: cuisinier qui apprête les légumes, les potages et les entremets;
- commis, boucher, rótisseur, grillardin, etc.

Le cuisinier talentueux, interresse et cons ciencieux peut acceder après quelques annees de pratique, a des postes tels que chef de partie, sous-chef, chef-exécutif

\section{La pátisserie-boulangerie}

Le cours de pattisserie-boulangerie a pour but de specialiser un éleve qui sera appelé à travailler au niveau des metiers de l'alimentation. II fabrique et cuit des patisseries, du pain et de la petite pátisserie. II est appelé également à confectionner les differentes decorations facilitant la vente des marchan. dises dans les centres d'hebergement ${ }^{\text {les }}$ restaurants et les pátisseries artisanales, commerciales et d'institutions. Le pätissier peut egalement travailler au niveau des laboratoires des grandes entreprises de l'alimentation. II fait les tests et verifications néces. saires à la bonne qulaite des produits.

Choix et contrôle des matières premières.

La carrière de pătissier s'echelonne comme suit: commis pâtissier, ouvrier pátissier, chef pâtissier ou chef de service, propriétaire d'un établissement.

\section{Le service de restaurant}

Le cours de service de restaurant a pour but de spécialiser un elève a servir des aliments et des boissons à la salle à manger, au bar et au service aux chambres d'un centre d"accueil ou de restauration tels que: hôtel, motel, auberge, restaurant ou traiteur.

La carrière de serveur s'échelonne cornme suit: commis, serveur, capitaine, maltre d'hôtel, directeur de restaurant. Les fonctions de barman, caviste et sommelier sont autant de métiers que peut exercer le diplömé en ser. vice de restaurant. 
A I'Université du Québec ả Montréal L'Université du Québec à Montréal se préoccupait du tourisme dès 1970 , au moment oủ s'ouvrait au Québec le Centre d'études du tourisme sous l'initiative de Pierre Courtemanche. Le C.E.T. lui-mème ne pré voyait pas faire de l'enseignement et de la recherche mais se presentait comme un ins trument de formation scientifique et technique dans ce domaine.

En 1971, un projet de centre de recherche en développement culturel accordant au tourisme une place de choix, fut déposé a la direction de la recherche de I'UOAM. Jugé prématuré à l'epoque, ce projet éclata finalement en différentes réalisations (I'Université du Québec à Trois-Rivières s'engageait en loisir, le département de sociologie de I'UQAM constituait un groupe de recherche sur la culture, etc.). Le tourisme, à cause de ses dimensions economiques omnipré sentes, fut repris par les sciences administratives et structuré en un programme de formation de 3 annẹes universitaires en 1976 .

Apres certaines consultations avec des representants de divers milieux touristiques, le baccalaureat en gestion et intervention touristiques de I'UOAM ouvrait ses portes en septembre 1978. II accueillait 60 étudiants. Contingenté, le programme n'admet que 90 nouveaux étudiants par année. Pré sentement, 220 personnes y poursuivent leurs études (dont un tiers à temps partiel) et 70 finissants sont sur le marche du travail (lou à la recherche d'emploi.). Trois professeurs y enseignent à plein temps, le reste de l'enseignement venant de plusieurs départements différents. Le programme actuel confere un diplôme de baccalauréat en administration des affaires (B.A.A.) avec spécialisation en tourisme. C'est dire que son orientation principale est en gestion. Les cours spécialisés en tourisme et les stages forment environ $35 \%$ du programme. Les étudiants peuvent choisir une concentration en analyse et planification ou en communication pour leur dernière annee de forma tion, s'ils ne veulent pas s'orienter prioritairement vers la gestion.

En janvier 1979, près de $50 \%$ des étudiants inscrits au programme venaient du marche du travail et étaient admis en vertu de l'ăge (22 ans et plus) et de l'expérience profes sionnelle pertinente $L$ ans de travail en tourisme). Aujourd'hui, ce pourcentage est descendu à $25 \%$.

\section{Autres niveaux}

Le tourisme lon devrait plutót specifier "les techniques en agences de voyage") s'enseignent aussi dans quelques écoles privees à Montreal. On y offre essentiellement des cours d'initiation al l'agence de voyage lune l'offre en trente heures, et l'autre en 99 heures... I. Le Centre de formation en tourisme offre en plus des cours de geographie touristique lles principales destinations touris- tiques). On peut compter enfin sur des stages de perfectionnement chez Air Canada afin d'acquérir des connaissances concernant surtout la tarification.

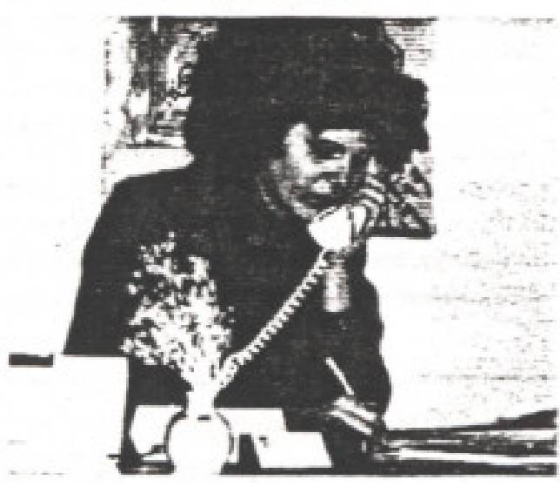

\section{L'après-formation}

\section{Le Regroupement des techniciens} en tourisme du Québec

(oủ mène la formation en tourisme?]

Le Regroupement des techniciens en tou risme du Québec a pour but de regrouper les techniciens en tourisme et les étudiants en techniques touristiques formes dans l'un des quatre collèges d'enseignement dispen sant le programme au Québec. Alors qu'existaient dejầ quelques associations regroupant les professionnels du tourisme et que leurs objectifs ne rejoignaient pas necessairement les nôtres, les techniciens en tourisme ont voulu creer une association qui prendrait à coeur et en main les intéréts et les besoins plus spécifiques des techniciens et étudiants en tourisme.

Ses principaux objectifs sont essentiellement:

- de faire connaitre et reconnaitre le technicien en tourisme dans le milieu touristique

- de promouvoir et representer l'intérét des membres auprès de toute institution oeuvrant dans le domaine touristique.

Pour parvenir à ses objectifs, le Regroupe ment des techniciens en tourisme du Qué bec rencontre à chaque année tous les étu. diants en techniques touristiques dans cha cun des collèges et maintient avec ceux-ci un lien étroit par le biais des directeurs etu diants. Aussi, I'an dernier, le R.T.T.Q a tenu un mini-colloque sous le titre "Le tourisme: une place à revendiquer ${ }^{* F}$. On retrouvait les ateliers suivants:

- La formation en tourisme

- La protection du technicien en tourisme dans son domaine d'emploi

- Le marché du travail: l'offre vs la demande

- le rôle du Regroupement des techniciens en tourisme du Québec dans le développement du tourisme au Québec.

Ces ateliers avaient pour but de tâter le pouls des membres quant au contenu d'un mémoire concernant la situation du technicien en tourisme en milieu de travail, rap. port qui devrait être presenté d'ici quelques mois.
De plus, le Regroupement des techniciens en tourisme du Québec publie bimestrielle. ment I'INFO-R.T.T.Q., bulletin d'informa tion et outil de communication pour les membres: le conseil d'administration y rend compte de ses activités et on y traite également d'actualité touristique.

Par sa représentation auprès du milieu tou ristique, le Regroupement des techniciens en tourisme se veut disponible à toujours faire connaïtre et reconnaitre le technicien en tourisme.

\section{L'Association technique du tourisme} (la "formation continue")

L'Association technique du tourisme du Québec est un organisme d'envergure provinciale qui contribue au développement du tourisme depuis sa création en 1975, en regroupant des intervenants de toutes les composantes du tourisme québécois.

Travailleurs, dirigeants, fonctionnaires, bené voles, professeurs, étudiants, corporations et institutions unissent leurs efforts vers des objectifs communs:

- Favoriser l'entraide et la collaboration entre les membres.

- Encourager l'étude, la recherche et la reflexion sur le phenomène du tourisme et ses composantes.

- Faciliter la transmission et la circulation de l'information scientifique et technique entre les institutions et les membres.

- Promouvoir et représenter les intérêts des membres auprès de toute institution oeuvrant entièrement ou partiellement dans le domaine du tourisme.

On devient membre pour s'intormer:

- En échangeant avec des gens intéres sants et en etablissant des relations enri. chissantes tant au point de vue personnel que protessionnel.

- En recevant les bulletins d' informations. en assistant a des rencontres.

Certaines activités permettent de se former

\section{- Congrés annuel}

Tout en etant le lieu de la rencontre annuelle des intervenants touristiques québécois, ce congrès fait le point sur l'évolution du tourisme au Québec.

\section{- Journées quebecoises du tourisme} Les Journees québecoises sont des colloques provinciaux ou internationaux sur des themes bien précis, tels: tourisme culturel, tourisme hivernal, tourisme d'affaires et des congres, tourisme rural, tourisme pour handicapes, etc.

\section{- Sessions de perfectionnement}

Des sessions d'une journée qui vous permettent d'acqueri de nouvelles connaissan ces dans diverses disciplines.

\section{- Bulletin d'information}

Les dernières nouvelles des membres, des éditoriaux, des commerciaux, un calendrier d'événements, les nouvelles régionales, des statistiques et encore des nouvelles.. 
L'Association technique du tourisme, il faut le souligner, vient egalement de faire parai tre un livre aux editions SODILIS: "Le tourisme, aspects theoriques et pratiques au Québec" sous la direction de Roger Nadeau. Par ces actions et son rôle de trait d'union, I'A.T.T. est donc un peu le lien, le carrefour des idees au niveau de la forma tion "continue" en tourisme au Québec.
Conclusion

Le tourisme est en pleine expansion, malgrề un certain falentissement dú ầ là situá tion économique générale dans le monde occidental. C'est un secteur d'activites qui. comme les sports et les activites de plein air et de loisir, va croitre encore considera blement. L'homme n'a pas fini de voyager, bien au contraire. Les modes, les moyens et les façons de voyager vont évoluer. Le champ d activites des travailleurs de l'indus. trie touristique couvre deja des domaines très varies, changeants, multi-disciplinaires: et ill va s'étendre encore bien davantage.

Dans l'ensemble, on peut dire que l'avenif du tourisme quebécois est entre bonnes mains. On peut ètre optimiste aussi, tout en sachant que les défis ne seront releves que par les plus dynamiques

\title{
La formation en tourisme et le marché du travail
}

\author{
La formation est-elle appropriee aux besoins du marché du travail? \\ Les diplómés en tourisme trouvent-ils facilement des emplois? \\ Quelle evaluation peut-on faire des programmes de formation dans cette perspective?
}

Trois points de vue sur cette question...et le debat est ouvert

\section{"Les maisons d'enseignement ne sont pas des centres de main-d'oeuvre"}

La formation en tourisme tente continuellement de s'adapter aux besoins du marche du travail, disons au "rythme normal" du système d'éducation public au Québec. Des efforts louables sont faits au niveau de la consultation et de la concertation, mais certains diront que cette adequation entre la formation et les divers secteurs du marche du travail s'effectue trop lentement.

Des exemples; les programmes relies à l'hờtellerie incluent des sta ges importants dans le milieu durant l'été ce qui favorise grandement l'apprentissage "sur le tas" et la connaissance des besoins reels et l'évolution dans le domaine. En tourisme, suite à la création des associations touristiques régionales au Québec, on a immédia tement ajouté le cours "Le tourisme régional au Quebec", pout répondre aux besoins des A.T.R.. On a crée de nouveaux cours auss pour s"adapter a l'evolution de la demande: "agrotourisme", "orga nisation de congres", "organisation de vovage", "animation. guidage", "promotion touristique", etc...

Mais depuis toujours, et c'est la mème chose dans presque tous les domaines, le champ d'activite des etudiants en tourisme s'étend de plus en plus. Les domaines sont varies, changeants, cycliques quelquefois. Les meilleurs parmi les nouveaux diplömés let ce n'est certes pas "le diplóme" qui prouve toute la competence nécessaire... peuvent choisir le domaine qui les interesse, et les autres doivent s'adapter à l'offre et a la demande. De toute façon, on peut dire aussi que les maisons d'enseignement ne sont pas des centres de main-d'oeuvre.

En général, on peut affirmer que de 50 à $70 \%$ des finissants se trouvent un emploi en tourisme apres leurs etudes. Certains continuent d'étudier, ou partent apprendre une autre langue à l'étranger, mais la plupart prennent plusieurs mois, mème jusqu'à un an, à se trouver un emploi. Depuis quelques années, ces emplois ne sont toutefois trop souvent que des projets subventionnes par les gouvernements fédéral et provincial, sinon ce sont des emplois "connexes": marketing, relation publique, guide accompagnateur, etc... Et on estime à environ $40 \%$ parmi ceux qui travaillent en tourisme, les techniciens qui se retrouvent dans une agence de voyage pour un certain temps.

Comme chacun le sait, le tourisme est une industrie et un secteur socio-économique qui n'emploie pas toujours des gens spécialisés, ceux qui ont cette comperence technique et professionnelle "certi. fiée" dans le domaine. On emploie quil l'on veut, selon les règles habituelles de la candidature idéale loủ le dynamisme, la personna lité et le bilinguisme priment bien davantage que les études....
Les diplômes ne sont pas tous officiellement reconnus. L'intégra tion au marché du travail se fait donc difficilement, lentement, de façon discontinue, ponctuelle, aléatoire, au rvthme des "dynamismes en place". Le marché du travail n'est pas complètement saturé mais les candidats doivent se faire à l'idee qu'ils accepteront des emplois connexes ou non reliés à leur champ de spécialisation.

De toute façon, cette formation "globaliste" en tourisme procure normalement à l'individu assez d'expertise, de maturite, et de confiance en soi pour "qu"il se prenne en charge" et qu"il réussisse à s'intégrer dans le monde du travail. Au pire let en blaguel, on réussit à former au moins de bons touristes...

\section{Que réserve l'avenir?}

Chaque institution tente de s'adapter aux besoins du marché. Des expériences sont tentees. On se dirige de plus en plus ves des cours "pratiques", des stages, des applications, des organisations diverses.

On étudie présentement la possibilité de spécialisation: deux ans de general, soit un tronc commun de cours identiques dans les institutions, et une année de spécialisation, soit en tourisme émetteur (agence de voyage) ou en tourisme réceptif laccueil, promotion, animation, amenagement...). On tentera peutetre un jour d'institutionnaliser aussi un stage pratique sur le marche du travail durant un semestre entier. Et quoi encore!

De toute façon, le tourisme est un domaine exigeant; et l'on constate que la grande majorité des diplömés dans ce domaine sont âgés seulement de 20 ans, ce qui implique que la plupart d'entre eux arrivent sur le marché du travail munis seulement de notions théoriques. La concurrence est très forte aussi: plusieurs diplömès en hôtellerie, en loisir, en récréologie, en aménagement forestier, sans compter les nombreux "conseillers en voyage" formes dans des écoles privees, viennent egalement joindre les rangs des "chercheurs d'emplois"' en tourisme.

Voila autant de bonnes raisons qui poussent les institutions à donner une formation intégrale de première qualité axée sur les dimensions du savoir, du savoir-faire et du savoir-être, afin que la clientele étudiante soit satisfaite et des plus aptes à répondre aux exi gences du marché du travail. Le defi est de taille. A l'image meme de la société, certains s'en sortent fort bien, d'autres sont déçus,... et plusieurs cherchent continuellement la place idéale, celle qui leur est due, celle qu'ils souhaitent ou celle qui leur convienne, dans ce "monde merveilleux" du tourisme.

André Bergeron

Professeur en techniques touristiques

Cegep de Granby 


\section{"On ne sait plus sur quel pied danser"}

Au bout des trois annees que dure la formation en techniques touristiques, les finissants se retrouvent le diplôme en poche, l'espoir au coeur, prèts à affronter le dur marché du travail. Alors commence la recherche d'emplois, les formulaires à remplir et la visite des centres de main-d'oeuvre oú on ne sait trop dans quelle catégorie vous classer. "Vous êtes guide touristique ou agent de voyages? Ni l'un ni l'autre, on est techniciens en tourisme."

Mais ceci n'est qu'un des pièges qui guettent le technicien en tourisme. Depuis plus de dix ans que se donne ce programme, on s'interroge toujours, à savoir si on doit spécialiser cette main-d'oeuvre ou bien au contraire lui donner une vision globaliste du tourisme. Méme les professeurs et les employeurs ne s'entendent pas la dessus. Ainsi, selon l'emploi auquel se destine l'étudiant, cela néces sitera une spécialisation, I"apprentissage de techniques spécifiques ou encore une connaissance générale du tourisme, du marketing et de la comptabilité. Par dessus-tout, une personne travaillant en tourisme se doit d'avoir de l'entregent, de l'initiative mais aussi beaucoup de patience pour arriver à se dénicher un poste convenable..

Tous ces facteurs font en sorte qu'on ne sait plus trop sur quel pied danser. Un tel emploveur vous dira que vous êtes trop spécialisé, un tel autre vous repondra que vous ne l'ètes pas assez, et quelque fois on vous dira que vous faites l'affaire mais malheureusement. vous savez, dũ aux coupures financières...

Un problème vient aussi du fait que dans trois des quatre colleges, on oriente les étudiants vers le tourisme receptif plutôt que vers

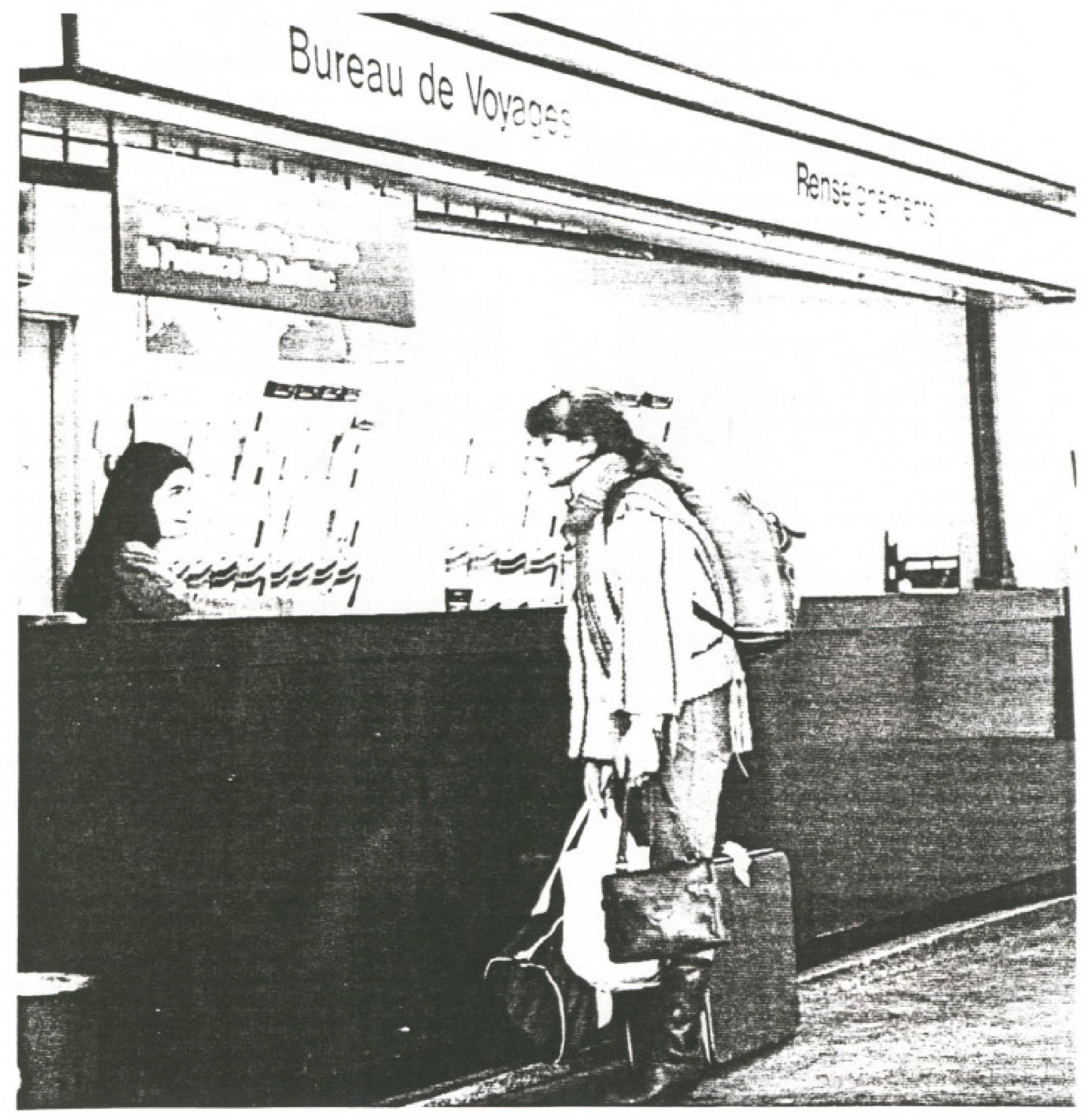


le tourisme émetteur. Mais en fait, examinons objectivement la situa tion, la demande semble plus grande en tourisme émetteur, les emplois sont plus nombreux, mieux rémunerés et comportant de meilleurs conditions de travaill, le domaine du tourisme émetteur est le plus lucratit. Quant au tourisme receptif, on en est encore aux entreprises sans but lucratif, toujours à la remorque d'une subvention, aux emplovês à petit salaire pour un projet subventionnè d'une duree de vingt semaines, un peu plus si on est chanceux. Ces projets n'aboutissent que rarement sur un emploi permanent.

Alors le technicien en tourisme chanceux se trouve un emploi pour quelques mois, mais il devient rapidement decu du manque d'avancement d'une part et d'autre part, des ressources financières limitees que lui procure son salaire.

Toute autre personne deviendrait comme lui, insecure, car entre un projet de quelques mois, l'assurance-chómage et un autre projet, le moral ne tient plus trop. On se decourage, on se cherche mainte nant un emploi dans un autre domaine, les jeunes pleins d'espoir. desappointés de n'avoir pas assez mangé de tourisme se tournent vers d'autres horizons. On les retrouve dans des domaines aussi divers que l'assurance, la comptabilité, le loisir, l'administration quand plusieurs ne retournent pas aux études.

Car, comme vous l'aurez sûrement remarqué, il n'est pas essentiel de posseder une formation en tourisme pour travailler en tourisme.
Ces illustres travailleurs en tourisme sont des geographes, récreologues, historiens, techniciens en loisir, diplómes en marketing, en administration, etc. La concurrence est grande et malgré l'anciennete du programme de techniques touristiques, on ne voit pas tel. lement de techniciens en tourisme dans des postes intèressants.

Nous ne croyons pas "qu"il faille être optimiste tout en sachant que les défis ne seront releves que par les plus dynamiques" reflète une grave lacune, celle du manque de concertation entre les instances gouvernementales, les directions des collèges, les profes: seurs, les étudiants et les techniciens en tourisme, mais surtout et par dessus tout une grande incohérence car on songe a former un certain nombre de techniciens en tourisme en sachant que seulement quelques-uns vivront de leur profession. Les techniciens en tourisme se retrouvent ainsi victimes de ce système et non pas favorisés par celui-ci

On ne doit pas former des techniciens en tourisme en esper ant que les défis ne soient releves que par les plus dynamiques mais bien pour que tous ces techniciens en tourisme contribuent ensemble à développer l'industrie touristique du Québec et fassent en sorte qu'elle devienne prospère car ne négligeons pas l'effet multiplica. teur du tourisme..

Michele L'Allier

Regroupement des techniciens du tourisme

\section{"Les diplômés doivent "vendre» leurs compétences"}

Par ses orientations et ses objectifs, le baccalaureat en gestion et intervention touristiques de I'UQAM est un programme d'études peu commun.

Habituellement, les formations universitiares de premier cycle en tourisme ne sont pas si ouvertes, polyvalentes et multidisciplinaires. Très souvent aussi, le tourisme n'est offert qu'au deuxième et troisième cycles, comme spécialisation, après l'acquisition d'un pre mier diplôme universitaire dans l'une ou l'autre des disciplines qui s'occupent fréquemment du tourisme léconomie, droit, géographie, administration, etcl.

L'originalité du programme d'études en tourisme de l'UQAM en fait a la fois sa force et sa faiblesse. Cette formation est particulièrement difficile à évaluer: d'une part, elle manque de points de comparaison; d'autre part, elle a été conçue pout un avenir à moven, sinon a long terme. Le potentiel du diplômé en tourisme de I"UQAM est considérable; il lui faudra cependant plusieurs annees de pratiques diverses pour arriver à un cadre et â un niveau d'intervention qui lui permettront de manifester toutes ses aptitudes acquises à IUniversite.

Les premiers finissants du module, ceux et celles qui ont reçu un diplöme depuis juin 1981, trouveront que les lignes précédentes sont trop optimistes. Selon un sondage-maison fait en janvier 1983 auprès de 35 des diplömés en tourisme de l'UQAM par madarne Patricia Oliveira et monsieur Abdellatif Aboukoubais, étudiants du module, plusieurs jugent que le programme n'est pas adapté aux exigences du marché du travail, qu'il est trop général, qu"il taudrait le spécialiser davantage en transport, hôtellerie, restauration, congrès, etc. Par ailleurs, $62 \%$ des étudiants actuels se disent satisfaits du baccalauréat en gestion et intervention touristiques... mais $91 \%$ ne sont pas certains que leur formation puisse répondre aux attentes du marché du travail. Finalement, une autre etude s'est adressé a un petit échantillon d'employeurs potentiels: le contenu et l'orientation du programme du baccalauréat de l'UQAM est très peu connu; 2 inter- venants sur 3 ne croient pas qu'il soit necessaire d'avoir un bache lier en tourisme au sein de leur organisme; très peu prévoient embaucher un diplobme universitaire en tourisme d'ici 5 ans; etc.

Bref, tel qu'orienté, le baccalauréat de l'UQAM semble un luxe. On lui reproche d'une part, d'ètre trop général et d'autre part, en le vou lant plus pratique, technique, immédiatement utile aux employeurs. on l'apparente encore plus à la formation collégiale, ce qu'on lui reproche aussi...

Je conclurai en exprimant un point de vue bien personnel à ce pro pos. Etudiant a l'Université Laval dans les années 1955-1960, jai assisté à la naissance des travailleurs sociaux plus tard, engagé en recherche en loisir, j'ai vu se professionnaliser l'éducation physique et la récrélogie. C'était vers 1965. A ce moment là, seule l'Université d'Ottawa formait quelques récréologues par année et ces pionniers devenaient ensuite des "hommes à tout faire" en loisir au Québec. Pourtant, à la mème date, j’assistai à un colloque à San Diego. Californie ou j"ai rencontre trois Ph.D. en "Park management" autour d'une table-rondel Nous avions quelques rattrapages baire au Québec.

La complexification croissante, signe distinctif de nos sociétés, récla mera ses doctorats en tourisme avant 10 ou 15 ans au Quebec. Entretemps, les responsables en place vont résister à cette profession. nalisation qui apparaît menaçante â certains. Entretemps aussi, les diplồmés universitaires devront s'armer de patience, faire de nom breux essais personnels, "vendre" leurs competences et poursui. vie des profils de carrière en zigzag. Comment faire autrement quand il s'agit de secteurs neufs? Comment faire la demonstration au maire d'une ville movenne, de 20,000 a 25,000 habitants, dans une belle région du Québec, qu'il a tout intêrêt à embaucher un bachelier en tourisme pour la mise en valeur de son milieu. Un jour, dans peu d'années peut-être. celá deviendra évident. D'ici là, il faudra que des pionniers, parmi nos diplômés, s'aventurent dans le bureau des maires pour les persuader. Cette audace ne s'enseigne pas. $f$

\section{Marc Laplante}

Directeur

Module de tourisme (UQAM) 